\title{
Clinicopathological and Immunohistochemical Features of Triple Negative Breast Cancer
}

\author{
Heng Chen ${ }^{1,2}$, Jinjun $\mathrm{Li}^{2 *}$, Wei Tan ${ }^{*}$ \\ ${ }^{1}$ Department of Ultrasound, Wuhan University of Science and Technology Hospital, Wuhan, China \\ ${ }^{2}$ Medical College, Wuhan University of Science and Technology, Wuhan, China \\ Email: *entry2003@126.com, ${ }^{\star} 2311449665 @ q q . c o m$
}

How to cite this paper: Chen, H., Li, J.J. and Tan, W. (2017) Clinicopathological and Immunohistochemical Features of Triple Negative Breast Cancer. Advances in Breast Cancer Research, 6, 93-99. https://doi.org/10.4236/abcr.2017.64008

Received: August 7, 2017

Accepted: September 5, 2017

Published: September 8, 2017

Copyright ( 92017 by authors and Scientific Research Publishing Inc. This work is licensed under the Creative Commons Attribution International License (CC BY 4.0).

http://creativecommons.org/licenses/by/4.0/

\section{cc) (i) Open Access}

\begin{abstract}
Objective: To investigate the clinicopathological and immunohistochemical features of triple-negative breast cancer. Methods: The clinicopathological and immunohistochemical data (Ki-67, CK5/6, EGFR, E-Ca, SAM, P53, P63, FAS) of 199 female patients who were treated for breast cancer in thyroid and breast surgery of Xiaogan Central Hospital from January 2015 to December 2016 was retrospectively analyzed by using spss22.0 statistical software and chi-square analysis. Results: Triple-negative breast cancer (replaced by TNBC below) and non-triple negative breast cancer (replaced by non TNBC below) in age, tumor size, lymph node metastasis, SAM, P53, P63 and FAS have no statistical difference $(P>0.05$, see Table 1$)$, while in WHO grade of invasive ductal carcinoma, KI-67, CK5/6, EGFR, E-Ca they have statistical differences $(P<0.05$, see Table 1$)$. The invasive ductal carcinoma WHO grade of TNBC is higher than that of non TNBC. It's positive rate of $\mathrm{Ki}-67, \mathrm{CK} 5 / 6$, EGFR (96.67\%, 58.33\%, 72.22\%) and negative rate of E-Ca (68.18\%) are higher than those of non TNBC $(75.74 \%, 29.03 \%, 18.92 \%, 30.38 \%)(P<0.05$, see Table 1$)$. Conclusions: The invasive ductal carcinoma WHO grade of TNBC is higher than that of non TNBC, while it's Ki-67, CK5/6, EGFR positive rate and the negative rate of E-Ca are significantly higher than those of non TNBC. The immunohistochemical index above is expected to become potential targets for the treatment of TNBC.
\end{abstract}

\section{Keywords}

TNBC, Clinicopathological Features, Immunohistochemistry

\section{Introduction}

Breast cancer is a more common malignancy in women. The number of new 
breast cancer patients is about 1.2 million each year. About 400,000 people have dead each year from breast cancer and the data increased by $2 \%$ to $3 \%$ per year [1]. At present, the medical research on breast cancer has entered the era of molecular level. Accurate grasp of breast cancer molecular typing characteristics is the necessary prerequisite of individual accurate treatment [2]. Although the treatment of breast cancer has been more mature, but the effect of endocrine therapy, targeted drug therapy and even chemotherapy is not very good for non TNBC due to the lack of expression of ER (estrogen receptor), PR (progesterone receptor), and HER-2 (human epidermal growth factor receptor 2) [3], which means that the need of finding other treatment breakthroughs is urgent. The clinicopathological and immunohistochemical data (Ki-67, CK5/6, EGFR, E-Ca, SAM, P53, P63, FAS) of 199 female patients who were treated for breast cancer in thyroid and breast surgery of Xiaogan Central Hospital from January 2015 to December 2016 was retrospectively analyzed below.

\section{Materials and Methods}

\subsection{Materials}

We selected 199 cases of breast cancer patients treated in Xiaogan City Center Hospital from January 2015 to December 2016 who underwent surgical treatment, postoperative pathology and immunohistochemistry. 199 cases of breast cancer patients were all female patients. The data of age, tumor diameter, lymph node metastasis, pathology, ER, PR, HER-2, KI-67, CK5/6, EGFR, E-Ca, SAM, P53, P63, FAS, Invasive ductal carcinoma patients WHO classification was collected as follows. One thing to note, the sample size was not same in all the parameter such as Expression of EGFR, P53, P63, SAM, FAS, E-Ca, because we found that there were some differences in the types of immunohistochemical markers detected in each tumor specimens during our retrospective study.

\subsection{Methods}

Collecting the eligible cases and completing the record of clinicopathological and immunohistochemical data (Ki-67, CK5/6, EGFR, E-Ca, SAM, P53, P63, FAS). HER-2 according to immunohistochemical staining of cells can be divided into $0,1+, 2+, 3+$. Among them $3+$ is positive, but $2+$ need to be further confirmed by FISH (HER-2 gene copy $<0.4$ is negative), and the rest is negative. Ki-67 positive cells $<14 \%$ is negative. For ER, PR, EGFR, P53, P63 and so on the positive cells $\geq 10.0 \%$ is positive, and $<10.0 \%$ is negative. According to the St Gallen consensus [4] published in year 2013, breast cancer is divided into four molecular types. The specific classification is as follows: ER and PR positive, HER-2 negative, Ki-67 < 14\% is Luminal A type; ER and/or PR positive, HER-2 negative, Ki-67 $\geq 14 \%$ and ER and/or PR positive, HER-2 positive, Ki-67 overexpression are Luminal B type; ER and PR negative, HER-2 positive is HER-2 positive type; ER, PR, HER-2 are negative called triple negative type. 


\subsection{Statistical Analysis}

Statistical analysis uses spss 22.0 statistical software and chi-square analysis. $P<$ 0.05 for the difference is statistically significant.

\section{Result}

Of the 199 women with breast cancer, 30 are TNBC, aged $42-80$ years old, with average of age 56 years old. Their tumor diameter are $1-6 \mathrm{~cm}$, with average 3.0 $\mathrm{cm}$. Lymph node metastasis rate is $46.67 \%$, and the number of invasive ductal carcinoma WHO classification I, II, III are respectively $0,1,9$. The positive rate of Ki-67, EGFR, P53, P63, SAM, FAS, CK5/6, are respectively 96.67\%, 72.22\%, $83.33 \%, 14.29 \%, 20.00 \%, 60.00 \%, 58.33 \%$. The negative rate of $\mathrm{E}-\mathrm{Ca}$ is $69.18 \%$. 169 cases are non TNBC, aged 32 - 81 years, with mean age of 51 years old. Their tumor diameter are $0.3-9 \mathrm{~cm}$, with average of $2.9 \mathrm{~cm}$. Lymph node metastasis rate is $51.48 \%$. The number of invasive ductal carcinoma WHO grade I, II, III are respectively $13,76,28$. The positive rate of Ki-67, EGFR, P53, P63, SAM, FAS, CK5/6, are respectively $75.74 \%, 18.92 \%, 63.41 \%, 40.63 \%, 36.36 \%, 86.68 \%$, $29.03 \%$. E-Ca negative rate is $30.38 \%$. There are no significant differences in age, tumor diameter, lymph node metastasis, SAM, P53, P63, FAS between the TNBC and non TNBC ( $P>0.05$, Table 1$)$, however the classification of invasive ductal carcinoma WHO, KI-67, CK5/6, EGFR, E-Ca are statistically different (all $P<0.05$, Table 1). $90 \%$ of TNBC invasive ductal carcinoma's WHO grade grade is III, while the number of non TNBC is $23.92 \%$, indicating that the TNBC invasive ductal carcinoma histological grade is higher than non TNBC. The TNBC's positive rates of Ki-67, CK5/6, EGFR and negative rate of E-Ca are higher than those of non TNBC (all $P<0.05$, Table 1 ).

\section{Discussion}

TNBC is ER (estrogen receptor), PR (progesterone receptor), and HER-2 (human epidermal growth factor receptor 2) all negative breast cancer, accounting for about $10 \%-22 \%$ of total breast cancer. This kind of breast cancer has become a hot spot in breast cancer research because of the poor results of chemotherapy, endocrine therapy and HER-2 targeted treatment, the rapid progress, aggressiveness, poor prognosis, common distant metastasis, poor prognosis and other features [5]. This paper retrospectively analyzed the clinical pathology and immunohistochemical data of 199 cases of female breast cancer patients treated with surgery in Xiaogan Central Hospital from January 2015 to December 2016. The clinicopathological and immunohistochemical data of TNBC and non TNBC are analyzed to compare the two to explore the specificity of TNBC.

At present, there is no conclusive conclusion in the comparison of clinical pathology between TNBC and non TNBC. One study reported that there are significant differences in age, tumor size, lymph node metastasis and so on, while in whether menopause, invasive ductal carcinoma grade and other aspects 
Table 1. Clinicopathological and immunohistochemical characteristics of triple-negative breast cancer and non-tri-negative breast cancer.

\begin{tabular}{|c|c|c|c|c|c|}
\hline Clinicopathological features & Total Number & TNBC & non TNBC & $x^{2}$ & $P$ \\
\hline \multicolumn{6}{|l|}{ Age } \\
\hline$\leq 35$ & 8 & 0 & 8 & \multirow{3}{*}{2.717} & \multirow{3}{*}{0.257} \\
\hline $35-60$ & 143 & 22 & 121 & & \\
\hline$\geq 60$ & 48 & 8 & 40 & & \\
\hline \multicolumn{6}{|l|}{ Diameter $(\mathrm{cm})$} \\
\hline$\leq 2$ & 74 & 8 & 66 & \multirow{3}{*}{3.226} & \multirow{3}{*}{0.199} \\
\hline $2-5$ & 96 & 19 & 77 & & \\
\hline$\geq 5$ & 29 & 3 & 26 & & \\
\hline \multicolumn{6}{|l|}{ Lymph node metastasis } \\
\hline no & 98 & 16 & 82 & \multirow{2}{*}{0.236} & \multirow{2}{*}{0.627} \\
\hline yes & 101 & 14 & 87 & & \\
\hline \multicolumn{6}{|l|}{$\begin{array}{l}\text { Invasive ductal carcinoma } \\
\text { WHO classification }\end{array}$} \\
\hline I & 13 & 0 & 13 & \multirow{3}{*}{18.294} & \multirow{3}{*}{0.000} \\
\hline II & 77 & 1 & 76 & & \\
\hline III & 37 & 9 & 28 & & \\
\hline \multicolumn{6}{|l|}{ Ki-67 } \\
\hline$<14 \%$ & 42 & 1 & 41 & \multirow{2}{*}{5.503} & \multirow{2}{*}{0.015} \\
\hline$\geq 14 \%$ & 157 & 29 & 128 & & \\
\hline \multicolumn{6}{|l|}{ EGFR } \\
\hline+ & 20 & 13 & 7 & \multirow{2}{*}{14.868} & \multirow{2}{*}{0.000} \\
\hline- & 35 & 5 & 30 & & \\
\hline \multicolumn{6}{|l|}{ P53 } \\
\hline+ & 62 & 10 & 52 & \multirow{2}{*}{1.069} & \multirow{2}{*}{0.301} \\
\hline- & 32 & 2 & 30 & & \\
\hline \multicolumn{6}{|l|}{ P63 } \\
\hline+ & 27 & 1 & 26 & \multirow{2}{*}{0.908} & \multirow{2}{*}{0.341} \\
\hline - & 44 & 6 & 38 & & \\
\hline \multicolumn{6}{|l|}{ SAM } \\
\hline+ & 25 & 1 & 24 & 0064 & \\
\hline - & 46 & 4 & 42 & 0.064 & 0.800 \\
\hline FAS & & & & & \\
\hline - & 10 & 2 & 8 & & \\
\hline+ & 47 & 3 & 44 & 3.232 & 0357 \\
\hline++ & 6 & 0 & 6 & 3.252 & 0.35 \\
\hline+++ & 2 & 0 & 2 & & \\
\hline E-Ca & & & & & \\
\hline+ & 62 & 7 & 55 & & \\
\hline - & 39 & 15 & 24 & 10.374 & 0.001 \\
\hline CK5/6 & & & & & \\
\hline+ & 32 & 14 & 18 & 6250 & 2012 \\
\hline - & 54 & 10 & 44 & 0.358 & 0.012 \\
\hline
\end{tabular}


the differences are not statistically significant [6]. But another study have shown that the differences of the two in age, tumor size, whether menopause and other aspects have no statistical significance, whlie in the lymph node metastasis, invasive ductal carcinoma grade the differences have statistical significance [7]. There is significant difference in the classification of WHO grade in invasive ductal carcinoma ( $P<0.05$, Table 1$)$, but there's no significant difference between the two groups in terms of age, tumor diameter and lymph node metastasis (All $P>0.05)$ in our report. It is reported that differences in this aspect may be due to genetic background, environmental factors or epidemiological differences [6].

$\mathrm{Ki}-67$ as an alternative marker of tumor proliferation, has become a marker of distinguishing Luminal A type and Luminal B type breast cancer, and among them the higher expression one is of Luminal B type, the other one is Luminal A type. To judge whether Ki-67 expression is high or not, we need to take the differences in the assessment of the organization into account [2]. They take $14 \%$ for the middle value in Xiaogan City Center Hospital. It has been found that Ki-67 expression in solid tumors is much higher than that in normal tissues and is associated with the development and metastasis of malignant tumors [8]. The high expression of $\mathrm{Ki}-67$ in breast cancer is a detrimental effect on the prognosis of breast cancer [9], and Ki-67-positive patients have shorter disease-free survival and overall survival [10]. This study shows that the positive rate of Ki-67 in the TNBC (96.67\%) is significantly higher than that in the non TNBC $(75.74 \%)$, which also confirms the characteristics of rapid development, common poor prognosis, distant metastasis and poor prognosis etc. of TNBC.

CK5/6 is a basal cell keratin, specifically expressed in the basal layer of epithelial cells. As the tumor cells usually retain specific skeletal midline silk protein expression of the original cell type. In the comparative study between CK5/6 and breast cancer prognosis, malignancy and so on, it's revealed that CK5/6 had basal cell type breast cancer origin characteristics. So it can detect tumor tissue CK5/6 to distinguish basal cell type breast cancer [11]. This study finds that TNBC CK5/6 positive rate $(58.33 \%)$ is significantly higher than non TNBC (29.03\%), indicating that TNBC mostly originates in the basal layer of breast tissue, which is consistent with Bertucci's study that $71 \%$ of TNBC shows basal phenotype and $77 \%$ of the basal-type breast cancer shows triple negative [12].

EGFR is a receptor for epithelial growth factor cell proliferation and signal transduction. It is one of the members of the epidermal growth factor receptor (HER) family and plays an important regulatory role in cell physiology. EGFR overexpression is common in epithelial tumors, whose cell proliferation is rapid and metastasis is common. One study shows that EGFR's positive expression rate of TNBC is of up to $78 \%$ [13], which is consistent with this study $(72.22 \%)$, and is significantly higher than that of non TNBC (18.92\%), indicating that EGFR plays an important role in the progression of TNBC and can be used as a potential target for the treatment of TNBC. In addition, its overexpression is ra- 
lated with the poor disease-free survival of TNBC [14]. Moreover, recent studies have shown that cetuximab as a EGFR target drug can increase the total remission rate of TNBC [15].

E-Cadherin (simply referred to as E-Ca) is a kind of adhesion protein. It is reported that E-Ca positive expression rate is of $32.28 \%$, which is similar to the results of this study (30.82\%), and its low expression can cause tumor cell adhesion decreased and promote its infiltration, local recurrence, lymphatic metastasis and distant spread [16]. Valproic acid (VPA) and Xylosiloxine (SAHA) can up-regulate the expression of E-Ca mRNA in endometrial carcinoma cells [17], and it is possible to help the treatment of TNBC by interfering with E-Ca expression.

\section{Conclusion}

The invasive ductal carcinoma WHO grade of TNBC is higher than that of non TNBC, while it's Ki-67, CK5/6, EGFR positive rate and the negative rate of E-Ca are significantly higher than those of non TNBC. The immunohistochemical index above is expected to become potential targets for the treatment of TNBC.

\section{References}

[1] Zheng, L.H. and Liu, R. (2015) Contralateral Breast Cancer Risk Factors. Chinese Journal of Cancer, 37, 241-243.

[2] Jiang, Z.F. (2015) Effect of Molecular Classification of Breast Cancer on Treatment. Chinese Journal of General Surgery (Electronic Version), 9, 12-15.

[3] Zhang, H., Wang, W., Tian, T., et al. (2016) Expression and Significance of Ezrin in Triple Negative Breast Cancer. Journal of Xi'an Jiaotong University: Medical Science, 37, 574-577.

[4] Goldhirsch, A., Winer, E.P., Coates, A.S., et al. (2013) Personalizing the Treatment of Women with Early Breast Cancer: Highlights of the St Gallen International Expert Consensus on the Primary Therapy of Early Breast Cancer 2013. Annals of Oncology, 24, 2206-2223. https://doi.org/10.1093/annonc/mdt303

[5] Yuan, Y.W. (2015) The Latest Treatment and Research Progress of Triple Negative Breast Cancer. Chongqing Medical, 44, 2279-2282.

[6] Tan, Q.Z. and Liu, D. (2014) Analysis of Clinicopathological Features and Prognostic Factors of Triple-Negative Breast Cancer and non-Tri-Negative Breast Cancer. Practical Journal of Cancer, 29, 1480-1482.

[7] Yang, M., Dai, D.L., Cui, G.Z., Chen, L. and Ding, M.J. (2015) Clinical Pathological Classification and Pathological Features of Triple-Negative Breast Cancer and Non-Triple Negative. Modern Journal of Integrated Traditional Chinese and Western Medicine, 24, 2713-2715.

[8] Shen, S.D., Chen, Z.R., Huang, Z., Xiao, G.F. and Lei, R.W. (2012) Expression and Significance of Ki67 in Different Molecular Types of Breast Cancer. The Practical Journal of Cancer, 27, 247-249.

[9] Han, J.G., Jiang, Y.D., Zhang, G.H., et al. (2011) Clinicopathologic Characteristics and Prognosis of Young Patients with Breast Cancer. Breast, 20, 370-372. https://doi.org/10.1016/j.breast.2011.02.011

[10] Weigel, M.T. and Dowsett, M. (2010) Current and Emerging Biomarkers in Breast 
Cancer: Prognosis and Prediction. Endocrine-Related Cancer, 17, 245-262. https://doi.org/10.1677/ERC-10-0136

[11] Chen, N., Wang, H., et al. (2014) Expression of CK5/6 in Triple-Negative Breast Cancer. Journal of Modern Oncology, 22, 1334-1336.

[12] Bertucct, F., Fineth, P., Cervera, N., et al. (2008) How Basal Are Triple-Negative Breast Cancers? International Journal of Cancer, 123, 236-240. https://doi.org/10.1002/ijc.23518

[13] Choi, J., Jung, W.H. and Koo, J.S. (2012) C1inicopathologic Features of Molecular Subtypes of Triple Negative Breast Cancer Based on Immunohistochemical Markers. Histology and Histopathology, 27, 1481.

[14] Prak, H.S., Jang, M.H., Kim, E.J., et al. (2014) High EGFR Gene Copy Number Predicts Poor Outcome in Triple-Negative Breast Cancer. Modern Pathology, 27, 1212-1222. https://doi.org/10.1038/modpathol.2013.251

[15] Baselga, J., Gomez, P., Greil, R., et al. (2013) Randomized Phase II Study of the Anti-Epidermal Growth Factor Receptor Monoclonal Antibody Cetuximab with Cisplatin versus Cisplatin Alone in Patients with Metastatic Triple-Negative Breast Cancer. Journal of Clinical Oncology, 31, 2586-2592.

https://doi.org/10.1200/JCO.2012.46.2408

[16] Liu, T., Shen, M., Wang, D., et al. (2014) Effects of Expression of MMP-9 and E-Cadherin in Triple-Negative Breast Cancer. Journal of Practical Oncology, 28, 19-23.

[17] Yin, T., Chen, X., Yu, H., et al. (2009) Effects of VPA and SAHA on Apoptosis of Endometrial Carcinoma Cells and E-Cad Gene Expression. Chinese Journal of Clinical Oncology, 36, 1120-1124. 